\title{
Interferometric adaptive optics for the National Ignition Facility
}

K. Baker, D. Homoelle, E. Stappaerts, C. Siders, C. Barty

August 12, 2011

SPIE Newsroom 
This document was prepared as an account of work sponsored by an agency of the United States government. Neither the United States government nor Lawrence Livermore National Security, LLC, nor any of their employees makes any warranty, expressed or implied, or assumes any legal liability or responsibility for the accuracy, completeness, or usefulness of any information, apparatus, product, or process disclosed, or represents that its use would not infringe privately owned rights. Reference herein to any specific commercial product, process, or service by trade name, trademark, manufacturer, or otherwise does not necessarily constitute or imply its endorsement, recommendation, or favoring by the United States government or Lawrence Livermore National Security, LLC. The views and opinions of authors expressed herein do not necessarily state or reflect those of the United States government or Lawrence Livermore National Security, LLC, and shall not be used for advertising or product endorsement purposes. 


\title{
Interferometric adaptive optics for the National Ignition Facility
}

\author{
K.L. Baker, D. Homoelle, E.A. Stappaerts, C.W. Siders and C.P.J. Barty
}

Great strides have been made in the pursuit of fusion energy as a relatively clean and essentially unlimited energy source over the past half century. One of the two approaches to achieving fusion energy, inertial confinement fusion, uses a large number of lasers to either directly compress a capsule containing Deuterium and Tritium or to indirectly convert the laser photons into x-rays on the wall of a Hohlraum and use these x-rays to compress a capsule filled with Deuterium and Tritium placed within the Hohlraum. The latter indirect drive ICF is currently being pursued by the newly commissioned National Ignition Facility, NIF, which is attempting to achieve ignition within the next couple of years. As we draw ever closer to solving the physics challenges associated with achieving fusion energy in the laboratory we begin to look closer at the daunting engineering challenges which must be solved in order for a commercial power plant driven by fusion energy to become a reality. One of those engineering challenges for inertial confinement fusion is the target tracking and beam correction for hundreds of laser beam lines which must occur. An ICF power plant will focus approximately 200 laser beams onto a Hohlraum, a cylinder with a diameter of $7 \mathrm{~mm}$ and a length of $14 \mathrm{~mm}$, containing a fuel capsule of Deuterium and Tritium, the fuel which will be compressed to generate the fusion energy. Hohlraums will be launched into the target chamber and their fuel capsule imploded at a rate of 10 hertz. The Hohlraums themselves will be injected into the target chamber at speeds of up to $200 \mathrm{~m} / \mathrm{sec}$ from standoff distances of perhaps $10 \mathrm{~m}$ with a requirement that each of the hundreds of lasers hit their respective positions on the Hohlraum's wall with positional accuracies of $\sim 100$ microns RMS. In the case of fast ignition inertial confinement fusion the positional accuracies of the beams can be as restrictive as 10 microns RMS and can require that multiple beam lines be phased together.

One approach to correct for the higher order beam aberrations, as well as to phase multiple beam lines together and to steer each of the beam lines is to use an interferometric adaptive optics, AO, system. When this interferometric wavefront sensor is used with a pixilated mirror it does not require a reconstruction of the measured wave-front and can therefore be run at correction speeds in excess of $10 \mathrm{khz}$ for systems exceeding a thousand subapertures, much faster than is possible with conventional AO systems.

A testbed was constructed at the Lawrence Livermore National Laboratory to assess the performance of such an interferometric AO system to simultaneously phase multiple apertures together, perform beam steering and also to correct for higher order aberrations within the beam lines before the system is implemented on the NIF for fast ignition experiments. For these fast ignition experiments the laser beam will be focused at the end of a fast ignition cone which is only 40 microns in diameter. To meet the energy requirements for the fast ignition experiments, four beam pairs must be phased together and the individual positioning of the beams at the end of the fast ignition cone be better than $10 \mu \mathrm{m}$ RMS. To change the pointing of a beam, a linear phase ramp is applied across the pixilated MEMS device. This causes the MEMS device to become a phase grating such that energy is taken out of the $m=0$ order and put into the higher orders of the grating as seen in Fig. 1a where four different levels of tilt are placed across the MEMS device. The inset graph shows the loss of Strehl ratio as a function of waves of tilt across the entire MEMS device. Phasing can be studied by placing microscope slides across half of the MEMS device and then measuring the phase and correcting for the piston error cause by the microscope slide. The resultant corrected far-field with these dephased apertures is shown in Fig. 1b with the inset graph showing the piston phase resulting when the microscope slide is placed across half of the aperture. The system achieved a corrected Strehl ratio of $\mathrm{S}_{\mathrm{r}}=0.76$.

This work performed under the auspices of the U.S. Department of Energy by Lawrence Livermore National Laboratory under Contract DE-AC52-07NA27344. 

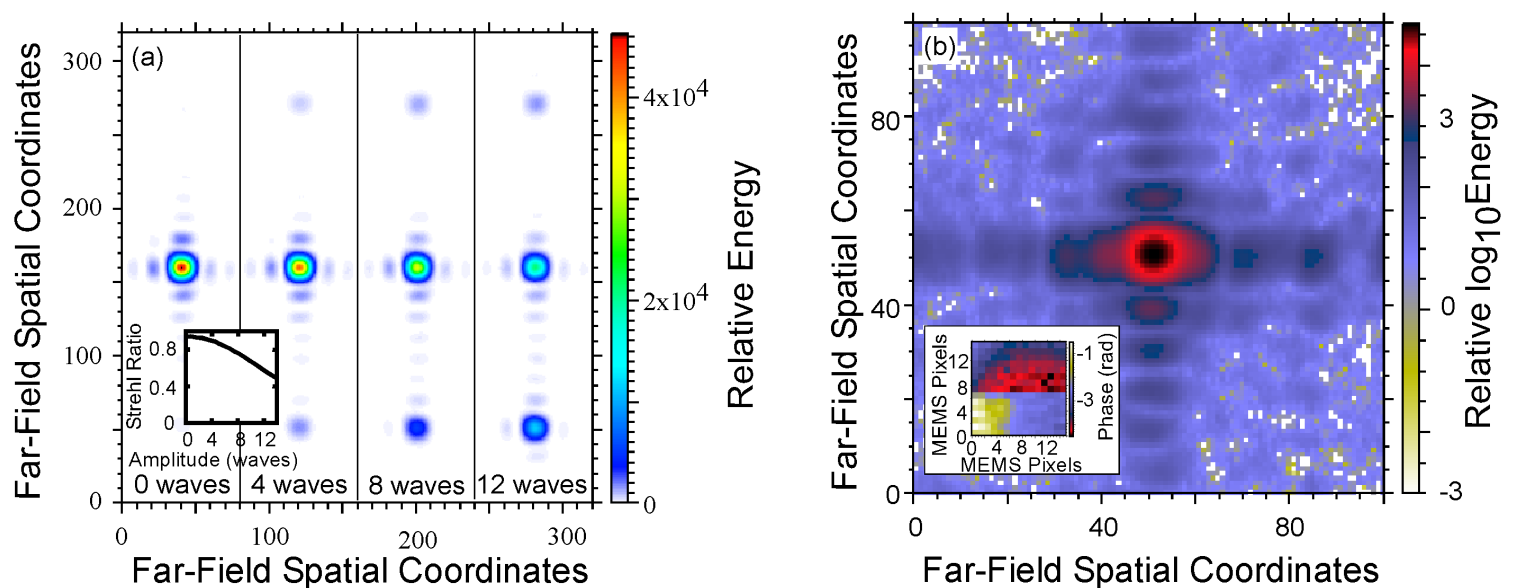

Fig. 1 Degradation in Strehl ratio due to an applied tilt error, Fig. 1a, and the corrected far-field after an application of a piston error across half of the aperture, Fig. $1 \mathrm{~b}$.

The testbed was also used to correct for a higher order aberration expected on the fast ignition beam lines. This phase, shown unwrapped in Fig. 2a, produced tip/tilt removed Strehl ratios of $S_{r}=0.087$. The corrected far-field achieved a Strehl ratio of $S_{r}=0.66$ as shown in Fig. 2b. The achieved Strehl ratios met the requirements for the fast ignition experiments and the system is now scheduled for implementation on the NIF.
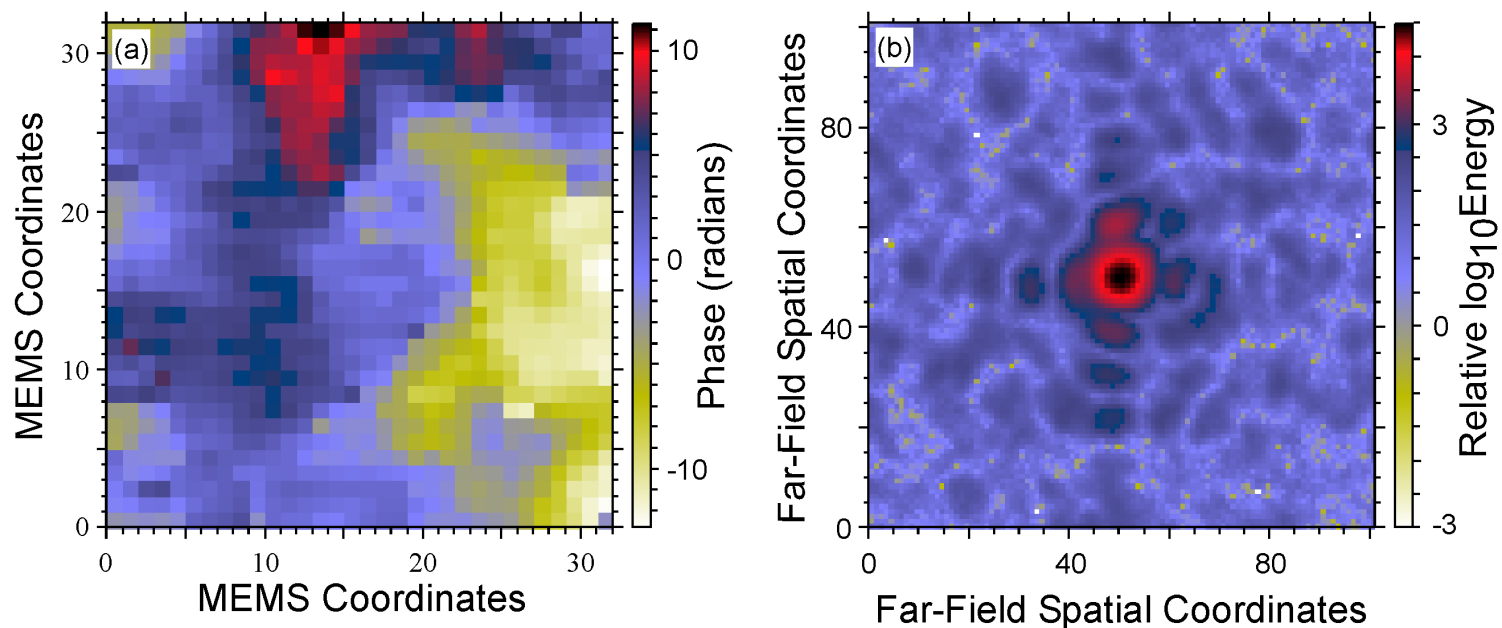

Fig. 2 Measurements examining higher-order phase errors across the aperture. The unwrapped phase is displayed in Fig. $2 \mathrm{a}$ and the corrected far-field intensity measurement is shown in Fig. $2 b$. 